\title{
Tratamento cirúrgico da doença de Ménière por meio de Shunts labirínticos: Passado e presente
}

\section{Surgical treatment of Meniere disease via labyrinth Shunt: Past and present}

\author{
Francisco Carlos Zuma e Maia', Alexandre Dolganov², Luiz Lavinsky³. \\ 1) Doutor em Otorrinolaringologia pela Universidade Federal do Rio Grande do Sul (UFRGS). Professor Convidado da Disciplina de Otorrinolaringologia da Faculdade \\ de Medicina e Cirurgia da Universidade Federal do Rio de Janeiro (UFRJ). \\ 2) Doutor em Neurologia. Professor Visitante da Pontifícia Universidade Católica do Rio Grande do Sul (PUCRS). \\ 3) Pós-Doutorado em Otorrinolaringologia. Professor da Faculdade de Medicina da UFRGS \\ Instituição: Centro de Pesquisa do Hospital de Clínicas de Porto Alegre. \\ Rua Ramiro Barcelos - 2350 - Bairro Rio Branco - Porto Alegre / RS - Brasil - CEP 90035-003 - Telefone: (+55 51) 2101-8232 \\ Endereço para correspondência: Francisco Carlos Zuma e Maia Rua Ipiranga, 95 - Sala 604 - Centro - Canoas / RS - Brasil - CEP: $92010-290$ - Telefone: (+55 51) \\ 3472-1820 - E-mail: zumaemaia@gmail.com \\ Artigo recebido em 10 de Fevereiro de 2010. Artigo aprovado em 29 de Maio de 2010
}

RESUMO

Introdução: As cirurgias dos shunts endolinfáticos empregadas para o tratamento da vertigem na doença de Ménière incapacitante permanece um tópico controverso. Portmann, em 1926, foi o primeiro a executar a cirurgia, incisando o saco endolinfático com o objetivo de diminuir a pressão endolinfática da orelha interna. Planejadas para criar uma fístula que conecte o espaço endo e perilinfático, as saculotomias foram descritas por FIck em 1964, por CoDy em 1969 e por Schunnecht (cocleossaculotomia) em 1982, entretanto foram paulatinamente abandonadas devido à alta incidência de perda auditiva. Uma nova e promissora opção cirúrgica dos shunts, em caráter experimental, é a utriculostomia, realizada por LAVINSKY em 1999.

Objetivo: Realizar uma revisão da literatura sobre as principais técnicas cirúrgicas dos shunts endolinfáticos empregados no tratamento da doença de Ménière, seus resultados e o que há de avanços no entendimento desses procedimentos. Método: Com base nos dados da literatura, são discutidas as principais técnicas cirúrgicas dos shunts endolinfáticos e sua racionalidade no tratamento $d a$ doença de Ménière incapacitante.

Conclusão: Existe muita controvérsia sobre a efetividade dos procedimentos cirúrgicos dos shunts endolinfáticos para o tratamento da doença de Ménière incapacitante, e muitos deles causam danos à orelha interna. A cirurgia experimental utriculostomia, portanto, parece-nos uma opção promissora. Futuras pesquisas poderão fornecer a resposta para essa questão.

Palavras-chave: doença de Ménière, vertigem, saco endolinfático.

\section{SUMMARY}

Introduction: The surgerys of the endolymphatic shunt are employed in the treatment of the vertigo of Meniere disease disabling, remains a controversial topic. Portmann in 1926, was the first to perform the surgery, incising the endolymphatic sac aimming the decrease of the endolymphatic pressure of the inner ear. Planned to create a fistula that connects the endo and perilymphatic space, the sacculotomy were described by Fick in 1964, by Cody in 1969 e by Schuknecht (cochleosacculotomy) in 1982, however they were gradually abandoned because of the high incidence of hearing loss. A new and promising surgery option of shunt, still experimental is utriculostomy, performed by LaVINSKY in 1999 .

Objective: Make a review of literature about the main surgical techniques of endolymphatic shunt, used in the treatment of Meniere disease, its results and the advance in the understanding of these procedures.

Methods: Based on the literature data, the main surgical techniques of endolymphatic shunt are discussed and its rationality in the treatment of Meniere's disabling disease.

Conclusion: There are a lot of controversy over the effectiveness of surgical procedures of endolymphatic shunt for the treatment of Meniere's disabling disease, and a lot of them damage the inner ear. The experimental surgery of utriculostomy, so it seems, a promising option. Future research can give an answer to this matter.

Keywords: Meniere disease, vertigo, endolymphatic sac. 


\section{INTRODUÇÃO}

A primeira opção de tratamento da doença de Ménière (DM) é medicamentosa, incluindo o uso de diurético, dieta pobre em sódio e beta-histina. Uma variedade de outras drogas podem ser usadas, incluindo antihistamínicos, benzodiazepínicos e corticoides. O tratamento cirúrgico somente é considerado quando a vertigem proveniente dessa condição progride a ponto de se tornar clinicamente incapacitante (1).

O alvo de todas as intervenções cirúrgicas é, primeiramente, reduzir o número e a severidade dos ataques agudos de vertigem e, secundariamente, melhorar ou estabilizar a perda auditiva e os zumbidos ou a plenitude aural. Para os pacientes que ainda permanecem incapacitados e com ataques de desequilíbrio depois de três a seis meses de terapia conservadora e envolvimento unilateral da doença, a intervenção cirúrgica pode ser considerada (2).

As intervenções cirúrgicas na DM podem ser divididas em dois grupos: conservadoras e não conservadoras da audição (1).

Os processos não conservadores incluem as labirintectomias e as neurectomias translabirínticas, que removem parcial ou totalmente o labirinto enfermo, e a inserção de aminoglicosídeo na orelha média para realizar uma labirintectomia química, que diminui a vertigem, mas pode resultar em perda auditiva (3).

Existe um outro grupo de técnicas não conservadoras da audição que atuam no sistema endolinfático distal, igualando as pressões e conectando os espaços endolinfático e perilinfático, atuando no nível do sáculo. Algumas são realizadas através da platina do estribo, outras através da janela redonda. Pela janela oval, temos a saculotomia, o procedimento da tacha e a saculocentese. Pela janela redonda, temos a "diurese osmótica" com $\mathrm{NaCl}$, a cocleostomia, a cocleossaculotomia, o shunt intracoclear e a diálise coclear. Existem ainda outras técnicas, que atuam em outras porções da cápsula labiríntica, como a criocirurgia, determinando uma fístula no canal semicircular lateral ou atuando no promontório com um shunt oticoperótico.

Entre as técnicas conservadoras da audição estão incluídas a cirurgia do saco endolinfático por descompressão simples, por descompressão e drenagem para a mastoide ou para o espaço subaracnoideo, implante de lâmina de Silastic ${ }^{\circledR}$ para a mastoide, por tubo capilar ou tubo capilar com câmara de fluido, shunt com válvula unidirecional sensível à pressão e, finalmente, shunt para o espaço subaracnóideo. Incluem-se, ainda, como medidas conservadoras, a neurectomia seletiva vestibular via fossa média, a neurectomia retrolabiríntica e neurectomia retrossigmóidea.

O procedimento mais utilizado na cirurgia do shunt é o do saco endolinfático. Apesar de essas cirurgias serem relativamente livres de complicações e tecnicamente de fácil realização, seus resultados são controversos: alguns autores afirmam não haver melhora da vertigem estatisticamente significativa em relação aos procedimentos cirúrgicos utilizados como placebos (4); já outros afirmam o contrário (5).

A tentativa de realizar procedimentos criando shunts endolinfáticos no nível do sáculo está em desuso, pelos altos índices de perdas auditivas (4).

Como se trata de uma área onde a otorrinolaringologia tem conceitos muitos variados e de difícil aplicabilidade, achamos que a ordenação conceitual sobre o tema e a respectiva discussão propiciariam uma contribuição consistente para os leitores. Para tal, criamos os seguintes objetivos:

1) Apresentar uma revisão bibliográfica das principais técnicas cirúrgicas dos shuntsendolinfáticos, ou seja, as mais empregadas no tratamento da DM.

2) Discutir seus resultados, destacando uma nova alternativa cirúrgica em fase experimental que atua no utrículo.

\section{REVISÃO DE LITERATURA}

\section{Shunts endolinfáticos no nível do saco endolinfático.}

O primeiro procedimento de shuntendolinfático foi realizado por Georges Portmann em 26 de fevereiro de 1926 (6). Como resultado de suas próprias pesquisas e da teoria proposta por Guild de que o fluxo endolinfático longitudinal ocorria em direção ao saco endolinfático (7), Portmann reconheceu que a DMé causada por um aumento de pressão no compartimento endolinfático da orelha interna. Ele imaginou que a cirurgia do saco endolinfático, desenhada para diminuir a pressão endolinfática no compartimento da orelha interna através da incisão do saco endolinfático, melhoraria os sintomas associados com a hidropisia (bydrops) endolinfática. Nesse procedimento, o saco endolinfático é aberto para o processo mastóideo. A cirurgia foi realizada com martelo e goiva ao invés de uma broca otológica, e nenhum microscópio foi utilizado. O saco era simplesmente incisado com uma pequena faca, teoricamente reservando a drenagem da endolinfa sob aumento de pressão em direção a mastoide. 
Com a maior utilização do microscópio e das brocas cirúrgicas, muitas modificações foram feitas à cirurgia original de Portmann com o intuito teórico de melhorar a drenagem da endolinfa. Em 1954, YAMAKAwA N NAITO removeram parte da parede medial do saco endolinfático para direcionar a drenagem do saco endolinfático em direção ao espaço subaracnóideo (8). Para evitar infecção e diminuição da drenagem, House, em 1962, criou um tubo para o shunt endolinfático-subaracnóideo. Para colocar esse tubo de polietileno, uma incisão era feita na parede medial do saco endolinfático, com o objetivo de criar uma fístula cirúrgica aberta. Subsequentemente, vários autores, incluindo SHEA (9) e Paparella e Hanson (10), entre outros (11-14), descreveram implantes de lâminas de Silastic ${ }^{\circledR}$ de drenagem, tubos ou material de cobertura, o qual era usado para a drenagem do saco em direção a mastoide, evitando assim que a drenagem entrasse no espaço subaracnóideo. Em 1978, ARENBERG et al. adicionaram uma válvula de direção única no tubo do shunt, como havia sido feito na Europa em 1975 por STAhle (15). Kitahara et al., na sequência, basearam sua drenagem na abertura do saco intramastoide, dobrando a parede lateral para fora e inserindo uma esponja de gelatina absorvível dentro do lúmen (16).

\section{Shunts endolinfáticos no nível do sáculo}

As cirurgias de shunts endolinfáticos foram planejadas com a finalidade de criar uma fístula através da qual o espaço endolinfático pode ser conectado ao espaço perilinfático, colocando o shunt interno na cápsula ótica. Essa fístula, teoricamente, faria cair à pressão ao drenar o fluxo para o espaço perilinfático de baixa pressão.

O primeiro procedimento realizado com esse intuito foi denominado de saculotomia. Em 1964, Fick, usando evidências histológicas da distensão do sáculo em pacientes com DM, propôs que a ruptura do sáculo distendido poderia equalizar a pressão entre a endolinfa e a perilinfa. O autor preconizou uma punctura na platina do estribo com uma agulha para fazer a ruptura na base do sáculo. CoDy modificou o procedimento de Fick, colocando uma tacha de aço inoxidável através da platina do estribo ( tack operation) (17). Ele teorizou que o sáculo poderia se romper na tacha de aço inoxidável cada vez que fosse se distender. Essa cirurgia era realizada através do meato auditivo externo, procedendo a um retalho timpanomeatal. Infelizmente, muitos pacientes sofreram progressivas perdas auditivas após serem submetidos a essas cirurgias. Schuknecht notou que os idealizadores desses procedimentos não haviam previsto que a parede sacular distendida estava usualmente aderida à platina do estribo na DM, desse modo causando, em longo prazo, uma dificuldade para gerar uma fístula com caráter eficiente e permanente (18).

\section{Cocleossaculotomia}

Esse procedimento foi proposto em 1982 por Schuknecht, com base em observações científicas. Ele observou, em modelos animais, que as fístulas entre os compartimentos de perilinfa e endolinfa na orelha interna eram compatíveis com a preservação da audição e demonstrou que a existência de tais fístulas ocorrem espontaneamente nos ossos temporais em humanos. SCHUKNECHT acreditava que essas fístulas causavam remissão dos sintomas clínicos da DM e que elas poderiam ser permanentemente criadas via fratura e ruptura do ducto coclear (6).

Indicada como tratamento de pacientes idosos com sintomas incapacitantes, que têm audição pobre, mas revelam boa função vestibular nas provas eletronistagmográficas (1), e pela rapidez e simplicidade do procedimento, a cocleossaculotomia é realizada sob anestesia local. Começa-se com a criação de um retalho timpanomeatal, através do qual o nicho da janela redonda é exposto. Um estilete de ângulo reto, de $3 \mathrm{~mm}$, é introduzido em todo seu comprimento através da membrana da janela redonda, em direção à janela oval. Nesse ponto, o instrumento avança através da lâmina espiral óssea e penetra no sáculo. O estilete é retirado, e a membrana da janela redonda é coberta com pericôndrio, gordura ou fáscia temporal. O retalho timpanomeatal é então recolocado.

\section{Shunt endolinfático no nível do utrículo}

O primeiro procedimento cirúrgico de shunt endolinfático atuando no utrículo foi proposto por LavinskY et al. em 1999 (19). A intenção desse procedimento esteve relacionada com a expectativa de um menor dano auditivo, tendo em vista que o utrículo não faz parte de uma unidade anatomofuncional com a cóclea, a exemplo do sáculo. Em estágio experimental, o método denominado de utriculostomia consiste em obter uma fístula permanente no labirinto membranoso, comunicando assim os espaços endolinfático e perilinfático no nível do utrículo. O procedimento foi posto em prática em ovelhas, através da janela oval, utilizando um microcautério por radiofrequência com programação de tempo de exposição e temperatura. O calor localizado, aplicado com agulha de $0,20 \mathrm{~mm}$, gera perda de substância. Conforme observado em exames histológicos, há uma regeneração na área tratada, formando uma frágil membrana que se comporta como uma válvula, rompendo-se em momentos de hidropisia endolinfática e, assim, comunicando os dois espaços e evitando uma permanente mistura iônica e suas conhecidas consequências.

A utriculostomia experimental foi realizada em 12 ovelhas no Hospital Veterinário da Escola de Medicina 
Veterinária da Universidade do Rio Grande do Sul, Porto Alegre, RS. Após a anestesia, a área endoperiauricular dos animais foi exposta e a membrana timpânica removida segundo a técnica descrita por House et al. (20), empregada em estapedectomias.

O promontório, as janelas oval e redonda, o nervo facial, o corda do tímpano, a apófise piramidal e o ligamento tensor do estribo foram expostos. Assim, o ligamento tensor do estribo foi seccionado e a disjunção da articulação incudoestapediana realizada. A supraestrutura do estribo foi fraturada, e toda a platina foi removida. Com uma broca de diamante, aproximadamente $2 \mathrm{~mm}$ da borda posterior da janela oval eram removidos em direção ao nervo facial. O alargamento da janela oval facilitava o procedimento de cauterização no utrículo.

A parede anterior do utrículo, parcialmente visível durante a otomicroscopia, era cauterizada utilizando-se um microtermocautério com ponteira de $0,2 \mathrm{~mm}$ por 0,5 segundos, a uma intensidade de $3,5 \mathrm{~W}$. A cauterização era repetida em três locais próximos um ao outro. A janela oval era fechada com tecido adiposo retirado de região próxima à incisão endoperiauricular e coberta com Gelfoam ${ }^{\circledR}$. O tímpano era reinserido, e o meato acústico externo tamponado com Gelfoam ${ }^{\circledR}$. Finalmente, a região era coberta com curativo compressivo.

Três meses após a cirurgia, o animal era sacrificado, e seu osso temporal era submetido a estudo histológico pertinente no laboratório de histopatologia dirigido por Michael Paparella (University of Minnesota, Minneapolis, EUA). Foi possível demonstrar a efetividade e a segurança da técnica proposta, com resultados positivos.

\section{DISCUSSÃO}

Os mecanismos de secreção e reabsorção realizados na escala média através do epitélio heterogêneo necessitam ser bem equilibrados para manter um constante volume de fluido. Falhas na manutenção desse equilíbrio resultarão na dilatação do compartimento endolinfático observado na DM. Tem-se também admitido que esse aumento da pressão endolinfática possa romper o labirinto membranoso e originar uma mistura entre a endolinfa, rica em potássio, e a perilinfa, pobre em potássio (21). O desequilíbrio do potássio impediria a despolarização e originaria perda transitória da função até que a membrana seja reparada e as relações sódio-potássio sejam restauradas.

Acredita-se que a vertigem resulte da sobreestimulação mecânica das células sensoriais devido à distensão das estruturas da orelha interna. Esta é causada pela disfunção do saco, lesado por fibrose, infecção ou processos autoimunes ou alérgicos. Outro mecanismo possível é a produção exagerada de glicoproteínas, que originaria um aporte excessivo de endolinfa no saco (21).

Dados da literatura indicam que pelo menos 85\% dos pacientes com DM terão uma boa resposta ao tratamento clínico (4). Porém, mesmo após 20 anos sofrendo da doença, 21\% dos pacientes permanecem com vertigem (22). Em um estudo prospectivo envolvendo 243 pacientes, houve aumento dos casos com ataques severos ou muitos severos (22), mostrando assim que, uma vez que a vertigem se torna incapacitante apesar do tratamento medicamentoso, o procedimento cirúrgico é indicado.

A descompressão do saco endolinfático é uma alternativa cirúrgica para aqueles pacientes que apresentaram resistência à terapia medicamentosa. Essa opção segura e conservadora do saco endolinfático pode ser realizada com ou sem a utilização de um shunt (1).

A lógica dessa cirurgia é muito discutida. Na base está o entendimento de que o shunt drena o excesso de endolinfa e alarga a luz do saco, ampliando a superfície de absorção, enquanto que a descompressão óssea reduz a pressão e aumenta o suprimento de sangue e as trocas (23).

Nos procedimentos cirúrgicos do saco endolinfático sem shunt, é realizada uma descompressão óssea ampla do saco, da área sigmóidea e da área do bulbo jugular, sem entrar no saco endolinfático propriamente dito. Resultados de seguimentos de longo prazo mostraram que a cirurgia é segura e eficiente para o controle da vertigem e para a estabilização da audição (24). As taxas de melhora vão de uma escala de 85 a 100\%, e a estabilização ou melhora da audição pode atingir quase 85\% (25).

A cirurgia do shunt do saco endolinfático é uma modificação da cirurgia sem shunt. Neste caso, um shunt é criado para o espaço subaracnóideo. O resultado do tratamento é comparável ao do shunt endolinfático mastóideo (26). Entretanto, em longo prazo, a presença de fibrose ou o fechamento da incisão do saco endolinfático podem prejudicar os resultados. A aplicação de mitomicina C intraoperatória no saco endolinfático incisado pode ser benéfica nesses casos (27), e a instilação de esteroides durante a cirurgia pode ajudar no controle da vertigem, na estabilização da audição e na diminuição do zumbido $(28,29)$. O controle da vertigem ocorre em 60-70\% dos casos na cirurgia do shunt do saco endolinfático (26). Apesar disso, tem-se sugerido que o impacto dos shunts do saco endolinfático sobre os sintomas dos pacientes com DM seja muito parecido com o obtido com placebo $(3,30)$, e alguns estudos dão suporte ao conceito de efeito placebo 
nessas cirurgias. A inserção de tubo de ventilação na membrana timpânica, a remoção completa extraóssea do saco endolinfático e a mastoidectomia cortical apresentam resultados similares ao procedimento do shunt do saco endolinfático (31-33). Entretanto, o estudo de Thomsen et al. contou com pequena amostragem e características estatísticas discutíveis. Pillsbury et al. (5) repetiram o estudo e obtiveram $87 \%$ de resultados favoráveis para o shunt, contra 47\% para o placebo (34).

Por outrolado, o procedimento cirúrgico em si pode ser crítico, porque a anatomia do saco endolinfático mostra variações, e o cirurgião pode ter dificuldades para encontrar e ampliar seu lúmen em alguns casos - essa condição pode afetar os resultados da cirurgia. A preservação da integridade do saco endolinfático também é importante. Uma cirurgia apropriada pode resultar em taxas de $90 \%$ de controle da vertigem (35).

A cirurgia do saco endolinfático pode ser realizada em crianças ou idosos, em pacientes com perda auditiva unilateral e em casos onde a DM coexiste com outras doenças, como fístulas, otite média e otosclerose. Ela pode ser realizada bilateralmente, entretanto não pode ser aplicada em condições vertiginosas outras que nas hidropisias endolinfáticas. A cirurgia pode resultar em 2\% de perda auditiva, o que pode ser atribuído mais a infecções ou inflamações do que ao procedimento cirúrgico em si (36). Embora os resultados da cirurgia do saco endolinfático sejam conflitantes, ela tem sido um dos procedimentos cirúrgicos mais comumente realizados na DM (35-37). Um estudo realizado na mesma instituição usando o guia da American Academy of Otolaryngology - Head and Neck Surgery mostrou que tanto a cirurgia do shunt do saco endolinfático para a mastoide quanto à descompressão do saco endolinfático são efetivas para os pacientes com DM (38). A cirurgia de descompressão do saco endolinfático, conforme descrita por PAPARELLA E SAJJADI, continua sendo a pedra angular da terapia cirúrgica para os pacientes com DM. Segundo os autores, o procedimento resistiu à prova do tempo, sendo um procedimento relativamente seguro, com um índice de menos de $2 \%$ de perda auditiva neurossensorial importante e menos de $1 \%$ de risco de paresia do nervo facial, controle importante (85\%) da vertigem durante 2 a 5 anos, com excelentes resultados também quando da revisão da cirurgia de aumento do saco endolinfático. Outros estudos, porém, têm demonstrado que a cirurgia do saco endolinfático não altera, a longo termo, o curso natural da vertigem na DM $(39,40)$. Em pacientes com DM há mais de 20 anos e que haviam sido submetidos à cirurgia do saco endolinfático, todos apresentaram longas, intensas e frequentes crises vertiginosas em comparação com os que não haviam realizado a cirurgia, sendo que $90 \%$ consideraram seus ataques severos ou muito severos (22).
A saculotomia inclui a drenagem do sáculo dilatado, com a resultante eliminação da hidropisia. Apesar das presentes modificações nas técnicas de saculotomia, a tack operation de CODY foi o mais popular dos procedimentos cirúrgicos realizados no sáculo. Segundo alguns autores, essa cirurgia pode ser uma opção quando o paciente tem uma perda auditiva no ouvido com hidropisia, pois quase $50 \%$ dos pacientes terão uma piora da audição, apesar do controle da vertigem com taxas acima de 60\% (41). Em recente estudo histopatológico (42) de osso temporal de pacientes submetidos a tack operation, foram observadas severa hidropisia endolinfática e mudanças degenerativas mais severas no órgão de Corti e nas células do gânglio espiral quando comparadas às da orelha contralateral, que não havia sido operada. Severa degeneração da mácula sacular também foi observada na orelha operada. Segundo os autores, esses achados estão em concordância com os resultados do estudo de hidropisia em modelo animal, onde as fístulas cirúrgicas endoperilinfáticas não foram suficientes para reduzir a hidropisia e ainda causaram mudanças degenerativas nas estruturas da orelha interna (43). Embora os autores (42) não tivessem como detectar a hidropisia e as mudanças nas estruturas da orelha interna antes e logo após a cirurgia, seus achados sugerem que esse tipo de cirurgia teve sucesso mais por aliviar a vertigem através da destruição das células ciliadas da mácula sacular do que pela resolução da hidropisia endolinfática. Os consideráveis danos ao órgão de Corti também demonstram os riscos de perda auditiva.

ScHuKnecht desenvolveu a cocleossaculotomia, criando uma fístula entre a endolinfa e a perilinfa com acesso via janela redonda. Seus resultados foram analisados em detalhe pelo próprio autor (SCHUKNECHT), idealizador do procedimento. Segundo o autor, a vantagem dessa cirurgia é sua simplicidade técnica e curto tempo de realização. Em sua revisão de 120 casos, uma melhora prolongada da vertigem foi atingida em $70 \%$ dos casos (44). Esses resultados são melhores do que os obtidos com qualquer outro procedimento de shuntendolinfático sacular, mas podem ser considerados pobres em comparação com a neurectomia do nervo vestibular, por exemplo, que atinge 94\% de controle da vertigem. Perdas auditivas parciais foram notadas em $25 \%$ dos pacientes, e surdez sensorioneural profunda ocorreu em mais de 10\%, em comparação com os menos de $5 \%$ associados a neurectomia vestibular. Embora outros estudos $(45,46)$ demonstrem controle de vertigem em mais de 80\% dos casos, a cocleossaculotomia não é advogada porque, em longo prazo, esse controle é pobre, e 30-80\% dos pacientes terão significativa perda auditiva (47). Atualmente, a cocleossaculotomia é raramente realizada, mas pode ser considerada em pacientes com DM idosos e cofóticos (48). 
A utriculostomia, alternativa cirúrgica em estágio experimental idealizada por Lavinsky et al. (19), consiste em obter uma fístula permanente no labirinto membranoso, comunicando os espaços endolinfático e perilinfático no utrículo. Tal objetivo foi posto em prática em ovelhas através da janela oval, utilizando um microcautério por radiofrequência com programa de tempo de exposição e temperatura.

Segundo o autor, o método se fundamenta no fato de que, quando se faz uma punção no sáculo, esta tende a apresentar um fechamento muito rápido, já que faz uma ruptura linear no labirinto membranoso. Ao contrário, o calor localizado, aplicado com agulha de $0,20 \mathrm{~mm}$, gera uma perda de substância, e observou-se histologicamente que há uma regeneração, formando uma frágil membrana que se comporta como uma válvula, rompendo-se em momentos de hidropisia endolinfática e, assim, comunicando os dois espaços e evitando uma permanente mistura iônica, com suas conhecidas consequências. Pela exiguidade da perfuração, esta tenderia a ficar fechada em momentos de intercrise da DM, gerando a comunicação somente em momentos de hidropisia endolinfática. Assim, a utriculostomia oferece nítidas vantagens no que se refere à permanente comunicação endo e perilinfática.

Na realidade, é provável que a utriculostomia criaria uma nova "válvula" em substituição ao mau funcionamento da válvula utrículo-endolinfática, também chamada de válvula de BAST (49). Embora sua função ainda seja incerta, na visão de alguns autores (50) ela se fecharia para prevenir maior perda de endolinfa no sistema utricular em casos de diminuição da pressão em todo sistema endolinfático - ou, como propôs ZECHNER (51), sua disfunção é que seria a causa da hidropisia endolinfática.

Nas observações pós-operatórias, apesar da pesada manipulação do vestíbulo, os autores relatam ausência de perdas significativas de equilíbrio e postura. Alguns animais apresentaram alterações no equilíbrio dinâmico, que desapareceram 24 horas após o procedimento, e nenhum nistagmo foi observado.

Por não atuar no sáculo, que tem íntima relação anatômica com a cóclea, esperam os autores poder demonstrar a existência de uma menor repercussão coclear na utriculostomia quando comparada com as saculostomias.

\section{COMENTÁRIOS FinAIS}

A literatura sobre cirurgias do shunt endolinfático apresenta muita controvérsia sobre sua efetividade no tratamento da DM incapacitante, sendo que muitos estudos inclusive relatam danos prejudiciais à orelha interna. A cirurgia experimental denominada utriculostomia aparece como uma nova e promissora opção para o tratamento cirúrgico da DM, por apresentar vantagens em relação às demais modalidades. Pesquisas adicionais devem se concentrar em avaliar esses aspectos.

\section{REFERÊNCIAS BIBLIOGRÁFICAS}

1. Lavinsky L. Tratamento cirúrgico do paciente vertiginoso. In: Lavinsky L (ed). Tratamento em otologia. Rio de Janeiro: Revinter; 2006, pp. 580-8.

2. Monsell EM. New and revised reporting guidelines from the Committee on Hearing and Equilibrium. American Academy of Otolaryngology-Head and Neck Surgery Foundation, Inc. Otolaryngol Head Neck Surg. 1995, 113:176-8.

3. Van Benthem PP, Giard JL, Verschuur HP. Surgery for Ménière disease. Cochrane Database of Systematic Reviews, 2009, Issue 4.

4. Storper IS, Michael E, Glasscock I. Internal and external endolymphatic shunts for Meniere's disease. In: Harris JP (ed). Meniere's disease. Amsterdam: Kugler; 1999. pp. 34954.

5. Pillsbury HC, Arenberg IK, Ferraro J, Ackley RS. Endolymphatic sac surgery. The Danish sham surgery study: an alternative analysis. Otolaryngol Clin North Am. 1983, 16:123-7.

6. Portmann G. Vertigo: surgical treatment by opening the saccus endolymphaticus. Acta Otolaryngol. 1927, 6:309-15.

7. Guild SR. The circulation of endolymph. Am J Anat. 1927, 39:57-81.

8. Yamakava K, Naito T. The modification of Portmann's operation for Meniere's disease (Yamakawa-Naito's operation). Med J Osaka Univ. 1954, 5:167-75.

9. Shea JJ. Teflon film drainage of the endolymphatic sac. Arch Otolaryngol. 1966, 83:316-9.

10. Paparella MM, Hanson DG. Endolymphatic sac drainage for intractable vertigo (method and experiences). Laryngoscope. 1976, 86:697-703.

11. Gardner G. Endolymphatic sac shunt operation in Meniere's disease. Trans Am Acad Ophthalmol Otolaryngol. 1975, 80:306-13.

12 Palva T, Ylikoski J, Paavolainen M, Holopainen E, Jauhianan T. Vestibular neurectomy and saccus 
decompression surgery in Meniere's disease. Acta Otolaryngol (Stockh). 1979, 88:74-8.

13. Miller GW, Welsh RL. Surgical management of vestibular Meniere's disease with endolymphatic mastoid shunt. Laryngoscope. 1983, 93:1430-40.

14. Spector GJ, Smith PG. Endolymphatic sac surgery for Meniere's disease. Ann Otol Rhinol Laryngol (Stockh). 1983, 92:113-8.

15 Stahle J. Ultrasound treatment of Meniere's disease. Long term follow-up of 356 advanced cases. Acta Otolaryngol (Stockh). 1985, 81:120-6.

16. Kitahara M, Kitano H. Surgical treatment of Meniere's disease. Am J Otol. 1985, 6:108-9.

17. Cody DTR, Simonton KM, Hallberg OE. Automatic repetitive decompression of the saccule in hydrops (tack operation): preliminary report. Laryngoscope. 1967, $77: 1480-501$.

18. Schuknecht HF. Pathology of Meniere's disease as it relates to the sac and tack procedures. Ann Otol Rhino Laryngol. 1977, 86:677-82.

19. Lavinsky L, Goycoolea M, Ganança MM, Zewtsch Y. Surgical treatment of vertigo by utriculostomy: an experimental study in sheep. Acta Otolaryngol (Stockh). 1999, 119:522-7.

20. House WF, Gardner G, Hughes RL. Middle cranial fossa approach to acoustic tumor surgery. Arch Otolaryngol. 1968, 88:631-41.

21. Benitez Del Rosario JJ, Escaméz JAL. Fisiopatologia de la enfermedad de Ménière. In: Ponencia oficial Del LX congreso nacional de la Sociedad Espanola de Otorrinolaringologia y Patologia Cervicofacial. 2009, 91-96.

22. Havia M, Kentala E. Progression of symptoms of dizziness in Ménière's disease. Arch Otolaryngol Head Neck Surg. 2004, 130:431-5.

23. Glasscock ME3rd, Jackson CG, Poc DS, Johnson GD. What I think of sac surgery in 1989. Am J Otol. 1989, 10:230-3.

24. Ostrowski VB, Kartush JM. Endolymphatic sac-vein decompression for intractable Ménière's disease: long-term treatment results. Otolaryngol Head Neck Surg. 2003, 128:550-9.

25. Gianoli GJ, Larouere MJ, Kartush JM, Wayman J. Sacvein decompression for intractable Ménière's disease: two year treatment results. Otolaryngol Head Neck Surg. 1998, 118:22-9.

26. Brackmann DE, Nissen RL. Ménière disease: results of treatment with the endolymphatic subarachnoid shunt compared with the endolymphatic mastoid shunt. Am JOtol. $1987,8: 275-82$.

27. Yazawa Y, Susuki M, KitanoH, Kitajima K. Intraoperative mitomycin C in endolymphatic sac surgery for Ménière's disease: a pilot study. ORL J Otorhinolaryngol Relat Spec. 1999, 61:184-94.

28. Kitahara T, Takeda N, Mishiro Y, Saika T, Fukushima M, Okumura S, et al. Effects of exposing the opened endolymphatic sac to large doses of steroids to treat intractable Ménière's disease. Ann Otol Rhinol Laryngol. 2001, 110:109-12.

29. Kitara T, Kubo T, Okumura S, Kitahara M. Effects of endolymphatic sac drainage with steroids for intractable Méniére's disease: a long-term follow-up and controlled study. Laryngoscope. 2008, 118(5):854-61.

30. Thomsen J, Bretlau P, Tos M, Johnsen NJ. Placebo effect in surgery for Ménière's disease: three-year follow-up. Otolaryngol Head Neck Surg. 1983, 91:183-6.

31. Thomsen J, Bonding P, Becker B, Stage J, Tos M. The non-specific effect of endolymphatic sac surgery in treatment of Ménière's disease: a prospective, randomized controlled study comparing "classic" endolymphatic sac surgery with insertion of a ventilation tube in the tympanic membrane. Acta Otolaryngol. 1998, 118:769-73.

32. Thomsen J, Kerr A, Bretlau P, Olsson J, Tos M. Endolymphatic sac surgery: why we do not do it. The nonspecific effect of a sac surgery. Clin Otolaryngol 1996, 21:20811.

33. Welling DB, Pasha R, Roth LJ, Barin K. The effect of endolymphatic sac excision in Ménière's disease. Am J Otol. 1996, 17:278-82.

34. Smith WC, Pillsbury HC. Surgical treatment of Ménière disease since Thomsen. Am J Otol. 1988, 9:39-43.

35. Huang TS. Endolymphatic sac surgery for Ménière disease: experience with over 3000 cases. Otolaryngol Clin North Am. 2002, 35:591-606.

36. Paparella MM, Fina M. Endolymphatic sac enhancement: reversal of pathogenesis. Otolaryngol Clin North Am. 2002, 35:621-37. 
37. Kerr AG. Emotional investments in surgical decision making. J Laryngol Otol. 2002, 116:575-9.

38. Brinson GM, Chen DA, Arriaga MA. Endolymphatic mastoid shunt versus endolymphatic sac decompression for Ménière's disease. Otolaryngol Head Neck Surg. 2007, $136: 415-21$.

39. Silverstein H, Smouha E, Jones R. Natural history vs surgery for Mèniére's disease. Otolaryngol Head Neck Surg. 1989, 100:6-16.

40. Filipo R, Barbara M. Natural history of Mèniére's disease: staging the patients or their symptoms? Acta Otolaryngol Suppl. 1997, 526:10-3.

41. MacDonald TJ, Cody TR. The sacculotomy procedure. Otolaryngol Clin North Am. 1995, 27:403-12.

42. Morita N, Cureoglu S, Paparella M. Tack operation for Ménière's disease: temporal bone histopathology case of the month. Otol Neurotol. 2009, 3. Epub ahead of print. doi: 10.1097/MAO.0b013e3181a527df.

43. Yazawa Y, Kitano H, Suzuki M, Kitanishi T, Kitajima K. Effects of endolymphatic-perilymphatic fistula on endolymphatic hydrops in guinea pig. Otolaryngol Head Neck Surg. 2000, 122:119-23.

44. Schucknecht HF. Cochlear endolymphatic shunt. Am J Otol. 1984, 5:546-8.
45. Montandon PB, Hausler RJ, Kimura RS. Treatment of endolymphatic hydrops with cochleosacculotomy. Clinical results and experimental findings. Otolaryngol Head Neck Surg. 1985, 93:615-21.

46. Dionne J. Cochleosacculotomy. J Otolaryngol. 1985, 14:59-61.

47. Giddings NA, Shelton C, O'Leary MJ, Brackmann DE. Cochleosacculotomy revisited. Long-term results poorer than expected. Arch Otolaryngol Head Neck Surg. 1991, 117:1150-2.

48. Kinney WC, Nalepa N, Hughes GB, Kinney SE. Cochleosacculotomy for the treatment of Ménière's disease in the elderly patient. Laryngoscope. 1995, 105:934-7.

49. Hofman R, Segenhout JM, Buytaert JA, Dirckx JJ, Wit HP. Morphology and function of Bast's valve additional insight in its functioning using 3D-reconstruction. Eur Arch Otorhinolaryngol. 2008, 265:153-7.

50. Barchor E, Karmody CS. The utriculo-endolymphatic valve in paediatric temporal bones. Eur Arch Otorhinolaryngol. 1995, 252:167-71.

51. Zechner G. Innenohrhydrops als Folge gestorter Endolymphzirkulation. Laringol Rhino Otol. 1980, 59:82933. 